\title{
How To Tackle RLN In Thyroid Surgery
}

Mr S.Ramachandran Locum ENT consultant,Mr V.R.Srinivasan ENT Consultant Arrowe park Hospital Wirral

Background-Over the years, thyroid surgery has gradually evolved into an ENT subspeciality from being a predominantly general surgical domain. Perfectly so, as ENT surgeons are most familiar with the anatomy of this area. Even in the current age of subspecilisation, an ENT surgeon is expected to be confident in handling neck conditions like lymph nodes, benign cysts or even benign salivary gland tumors and particularly, neck abscesses in an emergency. Thyroid surgery is gaining popularity amongst ENT trainees and perceived as a career option It is a very satisfying surgery, with a varied range of complexities from small volume benign nodules to huge retrosternal goitres. Preserving the Recurrent laryngeal nerve is the biggest challenge of a thyroid surgeon or even a deterrent to the weak hearted! Direct visualisation was the traditional approach and since the introduction of nerve monitors, it has gained popularity in various ENT surgeries and more recently in thyroid surgery.We would like to share our experience of over 700 thyroid surgeries performed by the senior author.

An uneventful surgery is any surgeon's desire! Complexities and complications are part of the deal and handling them successfully is the pleasure of operating. The recurrent laryngeal nerve is fairly consistent in its location, more so on the left side. It is found in the tracheoesophageal groove. Although, it can be slightly variable on the right, inferior thyroid artery is a very good landmark for identifying the RLN. Its not mandatory to identify the RLN, a subcapsular dissection preserving all non-thyroid tissue, without looking for the RLN is also an option.Middle thyroid vein,entry of RLN into larynx and inferior parathyroid are other useful landmarks to identify RLN(3).Even in case of large goitres, the location of the RLN isn't altered. It is good to befriend the nerves in the operating fields and this comes with practise. Adequate training is imperative to any surgery. In the context of thyroid surgery, the training is widely available, with large number of ENT units having a designated thyroid surgeon. The number of thyroid surgeries has increased over the years for various reasons-changing indications/guidelines, longevity of patients, reduced risks and hence better outcomes.

IONM works on the principle of detecting and amplifying electromyogenic potentials(4). In thyroid surgery we use a modified endotrache tube (see fig) with an electrode strip attached to it and externally connected to a monitoring device, this provides a continuous monitoring similar to mastoid and parotid surgery. A nerve stimulator probe is used to stimulate any structure which is perceived to be the nThere are a couple of pre-requisites a)the electrode must be in contact with the vocal cords b)the patient must be partially paralysed to retain muscle activity.

NICE published a guidance in $\angle U U 8$, in Which It states -IONM is an option used by some thyroid surgeons for revision surgeons and malignancies, perhaps it can be considered in routine thyroid surgeries.This is based on various non-randomised studies with substantial numbers. IONM is not fool proof, there's a possibility of false-negative results, in other words failure to identify the nerve which can be detrimental and result in nerve damage. Which was one of the arguments by the NICE advisory body and hence a false sense of reassurance to the surgeon. There is a role for it in training, it provides some sense of comfort to the trainee but more so to the trainer. 0 $4 \%$ incidence of permanent RLN palsy has been quoted in different non-randomised studies and transient palsy upto $9 \%$ at three months in the control group(direct visualisation or no identification). One of the large studies with 23,000 patients showed incidence of RLN palsy $3.5 \%$ for IONM and 3.7\% for direct visualisation(6). Interestingly, in some studies the incidence of RLN palsy in the group using IONM was more by $1 \%$ in both permanent and transient palsy, perhaps it was used mainly in complex cases like revision or malignancy. There's an ongoing Cochrane review, to minimise the bias of non-randomised studies

Our results- In our series, the senior author had 720 patients, in the age group 14-87yrs.M:F=1:4.5, 610 were benign, as expected. We had an overall permanent RLN palsy rate of $2.1 \%$, more common on the right side. IONM was used in all our patients.

Discussion- IONM is a simple monitoring device available in all ENT departments performing mastoid or parotid surgery. Its easy to setup, inexpensive and without any added risk to the patient. Permanent vocal cord palsy can be a very debilitating complication particularly in patients who rely on their voice for a livelihood. In the elderly swallowing poses a bigger problem. Although, the contralateral cord will compensate in due course, voice quality is altered and some patients bitterly complain of vocal fatigue. We also have the surgical options like augmentation if necessary. As with all complications, it is better prevented then treated. Even a regular thyroid surgeon can be caugh out by anatomical variations, its imperative to be attentive and have a thorough knowledge of anatomy. Any adjunct to minimise the risk of complications should be considered in the interest of the patient.

\section{Reference:}

1) JAMA otolaryngology H\&N Surgery 2016,Jun 1 584-87

2)P R Krakovitz et al,Intraoperative nerve monitoring during thyroidectomy-more complex than cost,

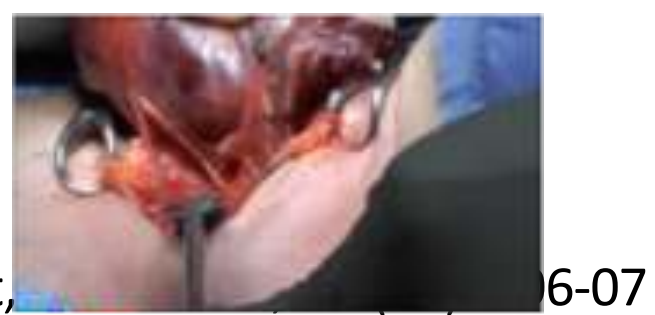

3) Chenling Shen et al,Routine exposure of RLN in thyroid surgery can prevent nerve injury,Neural Regeneration Research 2013 June $15 ; 8(17) 1568-1575$

4)ENT and Audiology news 26 issue,July/Aug 2017

5) Chan W.F et al- The role of IONM of RLN during thyroid surgery a comparative study of 1000 patients, Surgery 2006 Dec; 140(6) 866-72

6) Pisanu et al,Journal of Surgical Research,Dec 2017-Systematic review with meta-analysis of IONM of RLN versus direct visualisation 